\title{
Milli-Interacting Dark Matter Interpretation of the Direct-Search Experiments
}

\author{
Quentin Wallemacq \\ IFPA, AGO Department, University of Liège, Sart Tilman, 4000 Liège, Belgium \\ Correspondence should be addressed to Quentin Wallemacq; quentin.wallemacq@ulg.ac.be
}

Received 25 November 2013; Accepted 24 December 2013; Published 20 January 2014

Academic Editor: Konstantin Belotsky

Copyright (C) 2014 Quentin Wallemacq. This is an open access article distributed under the Creative Commons Attribution License, which permits unrestricted use, distribution, and reproduction in any medium, provided the original work is properly cited. The publication of this article was funded by $\mathrm{SCOAP}^{3}$.

\begin{abstract}
We reinterpret the results of the direct searches for dark matter in terms of milli-interacting dark particles. The model reproduces the positive results from DAMA/LIBRA and CoGeNT and is consistent with the absence of signal in the XENON100, CDMS-II/Ge, and LUX detectors. Dark atoms, interacting with standard atoms through a kinetic mixing between photons and dark photons and a mass mixing of $\sigma$ mesons with dark scalars, diffuse elastically in terrestrial matter where they deposit all their energy. Reaching underground detectors through gravity at thermal energies, they form bound states with nuclei of the active medium by radiative capture, which causes the emission of photons that produce the observed signals. The parameter space of the model is explored and regions reproducing the results at the $2 \sigma$ level are obtained for each experiment.
\end{abstract}

\section{Introduction}

Dark matter has been one of the most persistent enigmas in astrophysics since an invisible kind of matter was suggested in 1933 by Zwicky as an explanation to the missing mass between galaxies. Nowadays, the presence of dark matter is known at all cosmological scales and it is mostly believed that it is due to a unique species of collisionless particles, whose nature remains a mystery. One way to solve part of the problem is to observe directly these weakly interacting massive particles (WIMPs) in underground detectors. Such direct searches for dark matter have started in the late $1990 \mathrm{~s}$ and have led today to stunning results. The DAMA/LIBRA $[1,2]$ and CoGeNT $[3,4]$ experiments both have performed temporal analyses of their signals and confirmed the presence of an annual modulation of the event rates with statistical significances of $9.3 \sigma$ and $2.8 \sigma$, respectively. CRESST-II [5] and recently CDMS-II/Si [6] support these results with the observation of events in their detectors that cannot be due to background. On the other hand, XENON100 [7], CDMSII/Ge [8], and recently LUX [9] exclude any detection.

The current problem is that these experiments seem to come into conflict when their results are interpreted in terms of WIMPs producing nuclear recoils by colliding on nuclei in the detectors, although a more precise account for theoretical and experimental uncertainties could improve the status of WIMPs in that field. The tensions between experiments with positive results and the apparent incompatibility of the latter with experiments with negative results has led to considering other dark matter models that could provide new frameworks to reinterpret the data. Among these, mirror matter [10], millicharged atomic dark matter [11], O-helium dark atoms [12-14], and exothermic double-disk dark matter [15] propose interesting and varied mechanisms that can reconcile part of the experiments but always keep contradictions with the others. Light-mediator exchange [16] provides a viable mechanism which is able to explain the modulation effects, but its compatibility with the experiments with negative results is still uncertain. One common feature of all these scenarios is the high complexity of their dark sectors with respect to WIMPs, often reaching a phenomenology as rich as that of our ordinary sector.

The model presented here follows this trend and keeps some aspects of the above ones but presents new ingredients which aim at reconciling the experiments with positive results without contradicting those with negative results. The dark sector is composed of two new fermions both coupled to massless dark photons with opposite couplings and neutral 
dark scalars to which is coupled one of the two species via a Yukawa coupling. The oppositely charged dark fermions bind to form dark hydrogenoid atoms with standard atomic sizes. Such a dark matter candidate presents self-interactions on which constraints have been established from the Bullet Cluster and from halo shapes [17]. To avoid them, we follow [18] in which the self-interacting part of the dark sector is reduced to at most $5 \%$ of the total dark matter mass content of the galaxy, with the rest being realized by conventional collisionless particles presenting too weak interactions with standard particles to produce any recoil in underground detectors. The same kind of kinetic photon-dark photon mixing as in $[10,11]$ produces small effective couplings of the dark fermions to the standard photon, with the former behaving therefore like electric millicharges interacting with electrically charged standard particles. An additional mass mixing between the standard scalar $\sigma$ meson and the dark scalar creates an attractive interaction between one of the two dark fermions and the standard nucleons that are coupled to $\sigma$ in the framework of an effective Yukawa theory. The dark atoms interact sufficiently with terrestrial matter to lose all their energy between the surface and underground detectors, reaching them with thermal energies. There, dark and standard nuclei form bound states by radiative capture, causing the emission of photons that are the sources of the observed signals.

In [19], the model was introduced and a specific set of parameters that reproduced well the results from DAMA/LIBRA and CoGeNT and presenting no contradictions with XENON100 and CDMS-II/Ge was given. Here, the parameter space of the model is explored to determine the regions that reproduce DAMA/LIBRA and CoGeNT at the $2 \sigma$ level and to put upper and lower limits on the different parameters, always without contradicting the null results from XENON100 and CDMS-II/Ge, as well as the new constraint from LUX.

\section{Dark Sector}

In this model, the complex part of the dark sector is realized by two kinds of fermions, $F$ and $G$, of masses $m_{F}$ and $m_{G}$, interacting through a dark $U(1)$ gauge interaction carried out by dark massless photons $\Gamma$. In addition, the species $F$ exchanges dark neutral scalars $S$ of mass $m_{S}$ via a Yukawa coupling, which leads to the dark interaction Lagrangian:

$$
\mathscr{L}_{\mathrm{int}}^{\mathrm{dark}}=e^{\prime} \bar{\psi}_{F} \gamma^{\mu} A_{\mu}^{\prime} \psi_{F}-e^{\prime} \bar{\psi}_{G} \gamma^{\mu} A_{\mu}^{\prime} \psi_{G}+g^{\prime} \phi_{S} \bar{\psi}_{F} \psi_{F},
$$

where $\psi_{F}$ and $\psi_{G}$ are the fermionic fields of $F$ and $G, A^{\prime}$ and $\phi_{S}$ are the vectorial and real scalars fields of $\Gamma$ and $S,+e^{\prime}$ and $-e^{\prime}$ are the electric charges of $F$ and $G$, and $g^{\prime}$ is the Yukawa coupling of $F$ to $S$.

In order to produce nongravitational interactions between the standard and dark sectors, we postulate that the dark photons $\Gamma$ are kinetically mixed with the standard photons $\gamma$ and that the dark scalars $S$ are mixed with the neutral scalar mesons $\sigma$ via a mass term, with the mixing Lagrangian:

$$
\mathscr{L}_{\text {mix }}=\frac{1}{2} \widetilde{\epsilon}^{\mu \nu} \mathscr{F}_{\mu \nu}^{\prime}+\widetilde{\eta}\left(m_{\sigma}^{2}+m_{S}^{2}\right) \phi_{\sigma} \phi_{S}
$$

where $\mathscr{F}$ and $\mathscr{F}^{\prime}$ are the electromagnetic-field-strength tensors of the massless standard and dark photons, $\phi_{\sigma}$ is the real scalar field of the $\sigma$ meson, and $m_{\sigma}=600 \mathrm{MeV}$ [20] is its mass. $\widetilde{\epsilon}$ and $\widetilde{\eta}$ are the dimensionless parameters of the kinetic $\gamma-\Gamma$ and mass $\sigma-S$ mixings and are assumed to be small compared with unity.

In principle, the model contains seven free parameters, $m_{F}, m_{G}, m_{S}, e^{\prime}, g^{\prime}, \widetilde{\epsilon}$, and $\widetilde{\eta}$, but these can in fact be reduced to four. Indeed, only the products $\widetilde{\epsilon} e^{\prime}$ and $\tilde{\eta} g^{\prime}$ will be directly constrained by the direct-search experiments, which suggests to define them in terms of the charge of the proton $e$ and of the Yukawa coupling constant of the nucleon to the $\sigma$ meson $g=14.4$ [21]: $\widetilde{\epsilon} e^{\prime} \equiv \epsilon e$ and $\tilde{\eta} g^{\prime} \equiv \eta g$, where $\epsilon$ and $\eta$ are dimensionless mixing parameters. Moreover, the oppositely charged fermions $F$ and $G$ will bind to form hydrogenoid dark atoms where $F$ and $G$ will, respectively, play the roles of dark nucleus and dark electron, satisfying $m_{G} \ll m_{F}$. The Bohr radius of such atoms is given by $a_{0}^{\prime}=1 / m_{G} \alpha^{\prime}$, where $\alpha^{\prime}=$ $e^{\prime 2} / 4 \pi$, and gives another parameter that will be fixed to $1 \AA$ so that dark atoms have the same size as standard ones and can thermalize in the Earth before reaching the underground detectors, as will be specified in Section 3.3. As a result, the parameters of the model can be reexpressed as $m_{F}, m_{S}, \epsilon$, and $\eta$.

The dark particles $F$ will bind to nuclei in underground detectors and have therefore to be sufficiently massive to form bound states. For that reason, we will explore masses of $F$ between $10 \mathrm{GeV}$ and $10 \mathrm{TeV}$. The mass mixing term in (2) induces an attractive interaction between $F$ and nucleons with a range determined by $m_{S}^{-1}$. It cannot be too long ranged but it must allow the existence of nucleus- $F$ bound states of at least the size of the nucleus, so we will seek masses of $S$ between $100 \mathrm{keV}$ and $10 \mathrm{MeV}$. The model parameters that we will consider are therefore

$$
\begin{gathered}
10 \mathrm{GeV} \leq m_{F} \leq 10 \mathrm{TeV}, \\
100 \mathrm{keV} \leq m_{S} \leq 10 \mathrm{MeV}, \\
\epsilon, \eta \ll 1, \\
a_{0}^{\prime}=1 \AA .
\end{gathered}
$$

Note that the galactic dark matter halo could also be populated by dark ions $F$ and $G$, but [22] ensures that, if $\epsilon>9 \times 10^{-12}\left(m_{F, G} / \mathrm{GeV}\right)$, they have been evacuated from the disk by supernovae shock waves while galactic magnetic fields prevent them from reentering. This condition will clearly be satisfied by the parameters used to reproduce the results of the direct-dark-matter-search experiments and we can consider their signals to be fully due to dark atoms. 


\section{Interaction Potentials with Standard Matter}

Because of the mixings present in (2), the dark fermions $F$ and $G$ can interact with our standard particles. The kinetic $\gamma-\Gamma$ mixing induces small effective couplings $\pm \widetilde{\epsilon} e^{\prime}=$ $\pm \epsilon e$ to the standard photon for $F$ and $G$. The dark species can therefore interact electromagnetically with any charged standard particle with millicharges $\pm \epsilon e$. Similarly, the $\sigma-S$ mass mixing generates an effective coupling between $F$ and $\sigma$, making $F$ capable of interacting with any standard particle coupled to $\sigma$, that is, the nucleons in the framework of an effective Yukawa theory. In the nonrelativistic limit, these couplings give rise to interaction potentials between the dark and the standard particles.

3.1. Interactions of $F$ and $G$ Fermions with Nucleons and Electrons. At the elementary level, the kinetic $\gamma-\Gamma$ mixing produces a Coulomb interaction potential between the millicharged dark particles and the proton and the electron:

$$
V_{k}= \pm \frac{\epsilon \alpha}{r}
$$

where $k$ refers to kinetic and $\alpha=e^{2} / 4 \pi$ is the fine structure constant. The plus and minus signs stand, respectively, for the pairs proton- $F$ or electron- $G$ and proton- $G$ or electron- $F$.

Since the mass mixing parameter $\tilde{\eta}$ is small, the attractive interaction between $F$ and the nucleons is dominated by one $\sigma+S$ exchange, which gives

$$
V_{m}=-\frac{\eta\left(m_{\sigma}^{2}+m_{S}^{2}\right) \beta}{r}\left(\frac{e^{-m_{\sigma} r}-e^{-m_{S} r}}{m_{S}^{2}-m_{\sigma}^{2}}\right),
$$

where $m$ stands for mass and $\beta=g^{2} / 4 \pi=16.5$. Note that because $m_{S} \ll m_{\sigma}$, the potential (5) is essentially a Yukawa potential of range $m_{S}^{-1}: V_{m} \simeq-(\eta \beta / r) e^{-m_{S} r}$.

3.2. Interactions of F Fermions with Nuclei. Because of their interactions with nucleons, the dark fermions $F$ interact with atomic nuclei. Assuming that a nucleus of mass number $A$ and atomic number $Z$ is a uniformly charged sphere of radius $R=r_{0} A^{1 / 3}$ and volume $V=(4 / 3) \pi R^{3}$, the integrations of the elementary potentials (4) and (5) over its electric and nuclear charge distributions $\rho_{k}=Z e / V$ and $\rho_{m}=\mathrm{Ag} / \mathrm{V}$ give

$$
\begin{aligned}
V_{k}^{\mathrm{nucl}}(r) & =\int_{V}\left(\frac{V_{k}\left(\left|\vec{r}-\vec{r}^{\prime}\right|\right)}{e}\right) \rho_{k} d \overrightarrow{r^{\prime}} \\
& =\frac{\epsilon Z \alpha}{2 R}\left(3-\frac{r^{2}}{R^{2}}\right), \quad r<R \\
& =\frac{\epsilon Z \alpha}{r}, \quad r>R
\end{aligned}
$$

and $V_{m}^{\text {nucl }}(r)=\int_{V}\left(V\left(\left|\vec{r}-\vec{r}^{\prime}\right|\right)_{m} / g\right) \rho_{m} d \vec{r}^{\prime}$; that is,

$$
\begin{aligned}
& V_{m}^{\text {nucl }}(r<R) \\
& =-\frac{V_{0}}{r}\left[2 r\left(m_{\sigma}^{-2}-m_{S}^{-2}\right)\right. \\
& \quad+\left(R+m_{\sigma}^{-1}\right) m_{\pi}^{-2}\left(e^{-m_{\sigma} r}-e^{m_{\sigma} r}\right) e^{-m_{\sigma} R} \\
& \left.\quad-\left(R+m_{S}^{-1}\right) m_{S}^{-2}\left(e^{-m_{S} r}-e^{m_{S} r}\right) e^{-m_{S} R}\right], \\
& V_{m}^{\text {nucl }}(r>R) \\
& =-\frac{V_{0}}{r}\left[m_{\sigma}^{-2} e^{-m_{\sigma} r}\left(e^{m_{\sigma} R}\left(R-m_{\sigma}^{-1}\right)+e^{-m_{\sigma} R}\left(R+m_{\sigma}^{-1}\right)\right)\right. \\
& \quad-m_{S}^{-2} e^{-m_{S} r}\left(e^{m_{S} R}\left(R-m_{S}^{-1}\right)\right. \\
& \left.\left.\quad+e^{-m_{S} R}\left(R+m_{S}^{-1}\right)\right)\right],
\end{aligned}
$$

where nucl indicates nucleus, $\vec{r}^{\prime}$ is the position vector of a charge element in the nucleus, $V_{0}=3 \eta\left(m_{\sigma}^{2}+m_{S}^{2}\right) \beta /\left(2 r_{0}^{3}\left(m_{S}^{2}-\right.\right.$ $\left.m_{\sigma}^{2}\right)$ ), and $r_{0}=1.2 \mathrm{fm}$.

$V_{k}^{\text {nucl }}$ consists in a repulsive Coulomb potential outside the nucleus and in a harmonic potential, which is concave down, inside. Both are continuously connected at $r=R$ (as well as their first derivatives), with an inflection point at $r=R$ and a maximum reached at $r=0$, where the first derivative is zero.

$V_{m}^{\text {nucl }}$ corresponds to a finite attractive well, with a size of the order of $m_{S}^{-1}$, an inflection point at $r=R$ and tending to zero as $(-1 / r) e^{-m_{S} r}$ outside the nucleus.

The total nucleus- $F$ potential $V_{k}^{\text {nucl }}+V_{m}^{\text {nucl }}$ is therefore a negative attractive well at distances $r \lesssim m_{S}^{-1}$ that is continuously connected, together with its first derivative, to a positive potential barrier, coming from the repulsive Coulomb potential, at larger distances. As $r \rightarrow \infty, V_{m}^{\text {nucl }}$ rapidly tends to zero and the total potential is dominated by the Coulomb part. In order to reproduce the direct-search experiments, the depth of the well, mainly determined by the parameter $\eta$, will be of the order of $10 \mathrm{keV}$ while the barrier, which height depends on $m_{S}$ and $\epsilon$, will rise up to a few eV.

3.3. Interactions of Dark FG Atoms with Terrestrial Atoms. The galactic dark atoms interact, after hitting the surface of the Earth because of its motion in the dark matter halo, with terrestrial atoms under the surface. Because $m_{F} \gg m_{G}$, $F$ plays the role of a dark nucleus while $G$ is spherically distributed around it, so that the mass of the bound state $m_{F G}$ is almost equal to $m_{F}$. To model the atom-dark atom interaction, the dark atoms, as well as the terrestrial ones, are seen as uniformly charged spheres of charges $-\epsilon e$ and $-Z e$ and radii $a_{0}^{\prime}$ and $a_{0}$, respectively, where $Z$ is the atomic number of the terrestrial atom, with opposite point-like charges at their centers. The atomic radii are both fixed to $1 \AA$ to allow sufficient interaction for the dark atoms to lose all their kinetic energy by elastic collisions between the surface 
and the underground detectors and hence to reach them with thermal energies.

The atom-dark atom interaction potential is then the sum of the electrostatic interaction $V_{k}^{\text {at }}$ and the $\sigma+S$ echange $V_{m}^{\text {at }}$ between the dark nucleus $F$ and the atomic nucleus. $V_{k}^{\text {at }}$ is obtained by adding the contributions from the four pairs of crossed substructures: nucleus- $F$ (point-like-point-like, pure Coulomb repulsion), nucleus- $G$ distribution (pointlike-sphere, attractive of form (6)), electron distribution$F$ (sphere-point-like, attractive of form (6)), and electron distribution- $G$ distribution (sphere-sphere, obtained by integrating the form (6) over a uniformly charged sphere which center is separated by a distance $r$ from the center of the first one). This gives

$$
\begin{aligned}
& V_{k}^{\mathrm{at}}=\frac{\epsilon Z \alpha}{160 a_{0}^{6}}(-r^{5}+30 a_{0}^{2} r^{3}+80 a_{0}^{3} r^{2} \\
&\left.-288 a_{0}^{5}+\frac{160 a_{0}^{6}}{r}\right), \quad r<a_{0} \\
&= \frac{\epsilon Z \alpha}{160 a_{0}^{6}}\left(-r^{5}+30 a_{0}^{2} r^{3}-80 a_{0}^{3} r^{2}\right. \\
&\left.+192 a_{0}^{5}-\frac{160 a_{0}^{6}}{r}\right), \quad a_{0}<r<2 a_{0} \\
&=0, \quad r>2 a_{0},
\end{aligned}
$$

where the upper label at refers to atomic. Because the nucleus is here supposed to be pointlike, $V_{m}^{\text {at }}$ is simply obtained by multiplying (5) by the number $A$ of nucleons in the nucleus:

$$
V_{m}^{\mathrm{at}}(r)=-\frac{\eta\left(m_{\sigma}^{2}+m_{S}^{2}\right) A \beta}{r}\left(\frac{e^{-m_{\sigma} r}-e^{-m_{S} r}}{m_{S}^{2}-m_{\sigma}^{2}}\right) .
$$

The atom-dark atom electrostatic potential $V_{k}^{\text {at }}$ shows three parts as a function of the distance $r$ between the two centers. Each sphere appears neutral from outside because the positive charge at the center compensates exactly the negative charge distributed in the sphere, so that there is no interaction when they are completely separated $\left(r>2 a_{0}\right)$. As they merge $\left(a_{0}<r<2 a_{0}\right)$, the electrostatic potential becomes attractive due to the attraction between the nucleus of each sphere and the negatively charged distribution of the other one. When the nuclei enter simultaneously in the approaching spheres $\left(r=a_{0}\right)$, an inflection point occurs and the potential reaches then a minimum $\left(r=0.88 \AA\right.$ for $\left.a_{0}=1 \AA\right)$. As the centers continue to approach each other $\left(r<0.88 \AA\right.$ for $\left.a_{0}=1 \AA\right)$, the potential becomes repulsive due to the Coulomb repulsion between nuclei. The potential well appearing when the two atoms merge will have a depth of the order of $10^{-4}-10^{-3} \mathrm{eV}$ and will therefore not contain any bound state (or if any, not thermally stable), so that it will not contribute in the following to the formation of atom-dark atom bound states.

As $m_{S} \ll m_{\sigma}, V_{m}^{\text {at }} \simeq-(\eta A \beta / r) e^{-m_{S} r}$, which is a pure Yukawa potential. The total atom-dark atom potential $V_{k}^{\text {at }}+$ $V_{m}^{\text {at }}$ is therefore essentially equal to its electrostatic part $V_{k}^{\text {at }}$ for
$r<m_{S}^{-1}$, while the attractive part $V_{m}^{\text {at }}$ dominates at smaller distances.

\section{From Space to Underground Detectors}

4.1. Thermalization in the Terrestrial Crust. Due to its orbital motion around the Sun, which turns around the center of the galaxy, the Earth moves through the galactic dark matter halo. This results in a wind of dark atoms hitting the surface of the Earth throughout the year (however, according to [18], it is expected that the subdominant self-interacting species form a disk rotating around the galactic center, so that the incident flux on Earth might be different than in the halo assumption). A dark atom penetrates the surface and starts interacting with terrestrial atoms via the atomic potentials (8) and (9). As there is no stable bound state in the total atomic potential with the relatively light terrestrial atoms, the diffusions are purely elastic. If the elastic diffusion cross section is sufficiently large, then the dark atom can deposit all its energy in the terrestrial matter, assumed to be mainly made of silicon atoms with $Z_{\mathrm{Si}}=14$ and $A_{\mathrm{si}}=28$, before reaching an underground detector typically located at a depth of $1 \mathrm{~km}$.

A differential elastic diffusion cross section $d \sigma / d \Omega$ deriving from a two-body-interaction potential $V(\vec{r})$ can be obtained in the framework of the Born approximation via the Fourier transform of the potential: $d \sigma / d \Omega=$ $\left(\mu^{2} / 4 \pi^{2}\right)\left|\int d \vec{r} e^{-i \vec{K} \cdot \vec{r}} V(\vec{r})\right|^{2}$, where $\mu$ is the reduced mass of the two-body system and $\vec{K}$ is the transferred momentum. Here, from potentials (8) and (9) and in the center-of-mass frame of the silicon- $F$ system, we get

$$
\begin{aligned}
\left(\frac{d \sigma}{d \Omega}\right)^{\text {at }}= & \left(\frac{d \sigma}{d \Omega}\right)_{k}^{\text {at }}+\left(\frac{d \sigma}{d \Omega}\right)_{m}^{\text {at }} \\
& -\frac{4 \mu^{2} \epsilon \eta Z_{S i} A_{S i} \alpha \beta}{a_{0}^{6}}\left(\frac{m_{\sigma}^{2}+m_{S}^{2}}{m_{S}^{2}-m_{\sigma}^{2}}\right) \frac{I}{K^{8}} \\
& \times\left[\frac{1}{m_{\sigma}^{2}+K^{2}}-\frac{1}{m_{S}^{2}+K^{2}}\right],
\end{aligned}
$$

with

$$
\begin{gathered}
\left(\frac{d \sigma}{d \Omega}\right)_{k}^{\text {at }}=\frac{\mu^{2} \epsilon^{2} Z_{m S i}^{2} \alpha^{2}}{a_{0}^{12}} \frac{1}{K^{16}} I^{2}, \\
I=9\left(K^{2} a_{0}^{2}+1\right)+9 \cos \left(2 K a_{0}\right)\left(K^{2} a_{0}^{2}-1\right) \\
+12 \cos \left(K a_{0}\right) K^{4} a_{0}^{4}-18 \sin \left(2 K a_{0}\right) K a_{0} \\
-12 \sin \left(K a_{0}\right) K^{3} a_{0}^{3}+2 K^{6} a_{0}^{6}
\end{gathered}
$$

for the electrostatic interaction and

$$
\begin{aligned}
\left(\frac{d \sigma}{d \Omega}\right)_{m}^{\text {at }}= & 4 \mu^{2} \eta^{2} A_{m}^{2} \beta^{2}\left(\frac{m_{\sigma}^{2}+m_{S}^{2}}{m_{S}^{2}-m_{\sigma}^{2}}\right)^{2} \\
& \times\left[\frac{1}{m_{\sigma}^{2}+K^{2}}-\frac{1}{m_{S}^{2}+K^{2}}\right]^{2}
\end{aligned}
$$


for the $\sigma+S$ exchange, where $\mu=m_{F} m_{S i} /\left(m_{F}+m_{\mathrm{Si}}\right)$, where $m_{\mathrm{Si}}$ is the mass of a silicon atom, and $K=2 k \sin \theta / 2$, where $k=\sqrt{2 \mu E}$ is the initial momentum and $\theta$ is the deflection angle with respect to the collision axis.

For a dark atom to thermalize between the surface and an underground detector, we have to ensure that its penetration length does not exceed $1 \mathrm{~km}$. It is estimated by assuming a linear path of the dark atom through terrestrial matter:

$$
x=\int_{E_{\mathrm{th}}}^{E_{0}} \frac{d E}{|d E / d x|}<1,
$$

where $d E / d x$ is the energy loss per unit length in the frame of the Earth:

$$
\frac{d E}{d x}=n_{S i} \int_{\Omega} \Delta K\left(\frac{d \sigma}{d \Omega}\right)^{\mathrm{at}} d \Omega
$$

obtained by integrating over all diffusion angles. In (13), the integration is performed from the initial kinetic energy of the dark atom $E_{0}$ to the thermal energy of the medium $E_{\text {th }}=(3 / 2) T_{\text {med }}$, where $T_{\text {med }} \simeq 300 \mathrm{~K}$. In (14), $n_{S i}$ is the number density of atoms in the terrestrial crust and $\Delta K=$ $p^{2}(\cos \theta-1) / m_{S i}$ is the energy lost in the frame of the Earth for each collision with a silicon atom at rest in the terrestrial surface, expressed in terms of the momentum $p$ of each atom in the center-of-mass frame. It is clear that the linear path approximation is valid only when $m_{F} \gg m_{S i}$, but it gives in the other cases an upper limit on the penetration length of a dark atom through the Earth, which is of interest here.

4.2. Drift Down towards Underground Detectors. Once it has thermalized, a dark atom starts to drift towards the center of the Earth by gravity until it reaches an underground detector. The number density of dark atoms in the detector $n_{F}$ is determined by the equilibrium between the infalling flux at the surface and the down-drifting thermalized flux: $\left(n_{0} / 4\right)\left|\vec{V}_{h}+\vec{V}_{E}\right|=n_{F} V_{d}$, where $n_{0}\left(\mathrm{~cm}^{-3}\right)=3 \times 10^{-4} / m_{F}$ (TeV) is the local number density of dark atoms, $\vec{V}_{h}+\vec{V}_{E}$ is the superposition of the orbital velocity of the Sun around the galactic center $\vec{V}_{h}$ and of the Earth around the sun $\vec{V}_{E}$, and $V_{d}$ is the drift velocity of the dark atoms in the terrestrial matter once they have thermalized. Because of the orbital motion of the Earth around the Sun, $\left|\vec{V}_{h}+\vec{V}_{E}\right|$ is modulated in time: $\left|\vec{V}_{h}+\vec{V}_{E}\right|=V_{h}+V_{E} \cos \gamma \cos \left(\omega\left(t-t_{0}\right)\right)$, so that $n_{F}$ can be written as

$$
n_{F}=n_{F}^{0}+n_{F}^{m} \cos \left(\omega\left(t-t_{0}\right)\right)
$$

where $\gamma \simeq 60^{\circ}$ is the inclination angle of the Earth orbital plane with respect to the galactic plane, $\omega=2 \pi / T_{\text {orb }}$ is the angular frequency of the orbital motion of the Earth, $T_{\text {orb }}=1$ $\mathrm{yr}$ is the orbital period, and $t_{0} \simeq$ June 2 is the period of the year when the Earth and Sun orbital velocities are aligned. As $V_{d}=g / n\left\langle\sigma_{k}^{\text {at }} v\right\rangle$, where $g=980 \mathrm{~cm} / \mathrm{s}^{2}$ is the acceleration of gravity and $n \simeq 5 \times 10^{22} \mathrm{~cm}^{-3}$ is the number density of atoms in the terrestrial crust, the constant and modulated parts $n_{F}^{0}$ and $n_{F}^{m}$ can be expressed as

$$
\begin{gathered}
n_{F}^{0}=\frac{n_{0} n\left\langle\sigma_{k}^{\mathrm{at}} v\right\rangle}{4 g} V_{h}, \\
n_{F}^{m}=\frac{n_{0} n\left\langle\sigma_{k}^{\mathrm{at}} v\right\rangle}{4 g} V_{E} \cos \gamma .
\end{gathered}
$$

In these two expressions, $V_{h}=220 \times 10^{5} \mathrm{~cm} / \mathrm{s}, V_{E}=29.5 \times$ $10^{5} \mathrm{~cm} / \mathrm{s}$, and $n\left\langle\sigma_{k}^{\text {at }} v\right\rangle$ is the rate of elastic collisions between a thermalized dark atom $F G$ and terrestrial atoms, averaged over a Maxwellian velocity distribution at temperature $T_{\text {med }} \simeq$ $300 \mathrm{~K} . \sigma_{k}^{\text {at }}$ is obtained by integrating the differential cross section (11) over all diffusion angles and $v$ is the relative velocity between a dark atom and a terrestrial atom. Note that $\sigma_{k}^{\text {at }}$ dominates over $\sigma_{m}^{\text {at }}$ at low energies, so there is no need to consider the total cross section $\sigma^{\text {at }}$ here.

Arriving in the detector at room temperature, a dark atom still has to thermalize at the operating temperature. The latter is always lower than $300 \mathrm{~K}$, except for the DAMA detectors, which operate at room temperature. We will check that this second thermalization at the edge of the detector is realized over a distance much smaller than the typical size of the device and can therefore be considered as instantaneous.

4.3. Bound-State-Formation Events. In the active medium, the dark atoms undergo collisions with the constituent atoms. Because of the Coulomb barrier due to the electric repulsion between nuclei (potential (6)), most of these collisions are elastic, but sometimes tunneling through the barrier can occur and bring a dark nucleus $F$ into the region of the potential well present at smaller distance, due to the exchange of $\sigma$ and $S$ between $F$ and the nuclei of the detector (potential (7)). There, electric dipole transitions $E 1$ produce deexcitation of the system to low-energy bound states by emission of photons that can be detected, causing the observed signal. At this point, only the interaction between nuclei $V_{k}^{\text {nucl }}+V_{m}^{\text {nucl }}$ is therefore considered to calculate the capture cross section, since it dominates at small distance $(r \lesssim 1 \AA)$ and because the long-range part of the atom-dark atom potential $V_{k}^{\text {at }}+V_{m}^{\text {at }}$ is negligible and does not affect the initial diffusion eigenstate.

At thermal energies, to order $v / c$, only the partial $s$-wave of an incident plane wave on an attractive center is affected by the potential. Due to selection rules, direct $E 1$ transitions to final $s$-bound states are forbidden. It can also be shown that magnetic dipole and electric quadrupole transitions $M 1$ and $E 2$ to such final levels are not present [23], leaving only the possibility to capture $F$ in two $E 1$ transitions, first to a $p$-bound state and then to an $s$-bound state, corresponding, respectively, to levels at relative angular momenta $l=1$ and $l=0$ in the nucleus- $F$ potential $V_{k}^{\text {nucl }}+V_{m}^{\text {nucl }}$. The $E 1$ capture cross section of $F$ by a nucleus of charge $Z e$ and mass $m$ is then given by

$$
\sigma_{\text {capt }}=\frac{32 \pi^{2} Z^{2} \alpha}{3 \sqrt{2}}\left(\frac{m_{F}}{m_{F}+m}\right)^{2} \frac{1}{\sqrt{\mu}} \frac{\left(E-E_{f}\right)^{3}}{E^{3 / 2}} D^{2},
$$


where $\mu=m_{F} m /\left(m_{F}+m\right)$ is the reduced mass of the nucleus$F$ system, $E$ is the total incident energy in the center-of-mass frame, $E_{f}$ is the binding energy of the lower bound state at $l=1$, and $D=\int_{0}^{\infty} r R_{f}(r) R(r) r^{2} d r$, where $R$ and $R_{f}$ are the radial parts of the eigenfunctions of energies $E$ and $E_{f}$, with $r$ being the relative distance between $F$ and the nucleus.

It is important to note here that each capture event will give rise to the emission of two photons. For the events to be seen as single-hit events, as stated by DAMA, one will require that the first emitted photon with the greatest possible energy, corresponding to the $E 1$ capture from the continuum at $E \sim 10^{-2} \mathrm{eV}$ to the lower $p$-state $E_{f}$, has an energy below the threshold $E_{\text {threshold }}$ of the considered experiment. In other words, we will have $\left|E_{f}-E\right| \simeq\left|E_{f}\right|<E_{\text {threshold. }}$. The second emitted photon, corresponding to the $E 1$ transition from a $p$-state $E^{l=1}$ to an $s$-state $E^{l=0}$, will have an energy beyond the threshold; that is, $\left|E^{l=0}-E^{l=1}\right|>E_{\text {threshold }}$.

Thermal motion in a detector at temperature $T$ made of nuclei $N$ gives rise to collisions between $N$ and $F$ species and hence to the event counting rate per unit volume:

$$
R=n_{F} n_{N}\left\langle\sigma_{\text {capt }} v\right\rangle
$$

where $n_{F}$ and $n_{N}$ are the number densities of $F$ and $N$ in the detector and $\left\langle\sigma_{\text {capt }} v\right\rangle$ is the thermally averaged capture cross section times the relative velocity. Using Maxwellian velocity distributions at temperature $T$ in the frame of the detector, passing to center-of-mass and relative velocities $\vec{v}_{C M}$ and $\vec{v}$ and performing the integral over the center-of-mass variables, we get

$$
R=8 \pi n_{F} n_{N} \frac{1}{(2 \pi T)^{3 / 2}} \frac{1}{\mu^{1 / 2}} \int_{0}^{\infty} \sigma_{\text {capt }}(E) E e^{-E / T} d E .
$$

Given the modulated form (15) of the number density of $F$, one gets a modulated expression for the event rate:

$$
R=R^{0}+R^{m} \cos \left(\omega\left(t-t_{0}\right)\right)
$$

The constant and modulated parts $R^{0}$ and $R^{m}$, when expressed in counts per day and per kilogram $(\mathrm{cpd} / \mathrm{kg})$, are given by

$$
\begin{aligned}
& R^{0}=C n_{F}^{0} \int_{0}^{\infty} \sigma_{\text {capt }}(E) E e^{-E / T} d E, \\
& R^{m}=C n_{F}^{m} \int_{0}^{\infty} \sigma_{\text {capt }}(E) E e^{-E / T} d E,
\end{aligned}
$$

with (note that a factor of $\pi$ was missing in $C$ in [19] and has been corrected here)

$$
C=7.54 \times 10^{11} \frac{\mathrm{QtN}_{A v}}{M_{\mathrm{mol}}} \frac{1}{(2 \pi T)^{3 / 2}} \frac{1}{\mu^{1 / 2}},
$$

where $Q=1000 \mathrm{~g}, t=86400 \mathrm{~s}, N_{A v}=6.022 \times 10^{23}$, and $M_{\mathrm{mol}}$ is the molar mass of the active medium of the detector in $\mathrm{g} / \mathrm{mol}$.

An important feature of the model is its reinterpretation of the results of the direct-search experiments in terms of bound-state-formation events emitting photons that produce the observed signals. This is in opposition to the common scenario where WIMPs colliding on nuclei at velocity $220 \mathrm{~km} / \mathrm{s}$ produce nuclear recoils: here, the thermal energies in play in the detectors are insufficient to create such recoils, and the emitted photons cause electron recoils. In experiments that do not discriminate between these two kinds of recoils, as DAMA/LIBRA and CoGeNT, the reinterpretation is straightforward. In experiments with a discrimination power, the present dark atoms are good candidates if the results are negative, as it is the case for XENON100, LUX, and CDMS-II/Ge. Indeed, even if the bound-state-formation events cannot be naturally suppressed, the remaining events will be interpreted as backgrounds and rejected. Further studies have to be performed in the case of discriminative experiments with positive results, as CRESST-II and CDMS$\mathrm{II} / \mathrm{Si}$, to find if it is possible that the observed nuclear recoils may be misinterpreted bound-state-formation events occurring near the edge of those detectors.

\section{Exploring the Parameter Space}

5.1. Reproduction of the Results from DAMA and CoGeNT. The DAMA/LIBRA and CoGeNT experiments observe integrated modulation amplitudes $\widetilde{R}_{\mathrm{DAMA}}^{m}=(0.0464 \pm$ $0.0052) \mathrm{cpd} / \mathrm{kg}$ and $\widetilde{R}_{\mathrm{CoGeNT}}^{m}=(1.66 \pm 0.38) \mathrm{cpd} / \mathrm{kg}$ in the energy intervals (2-6) $\mathrm{keV}$ and (0.5-2.5) keV, respectively.

As a first approximation and for simplicity, the signal is supposed to be made of one monochromatic line of energy $\Delta E=E_{g}-E_{f}$, where $E_{g}$ is the ground state at $l=0$, falling within the detection range (it would be interesting to reproduce the observed energy spectra by taking into account the different possible transitions from the $p$-states to the $s$ states).

The 4-dimensional parameter space of the model is explored separately for DAMA and CoGeNT in order to reproduce the observed rates and energy intervals at the $2 \sigma$ level, which gives corresponding regions for each experiment. We use the isotopes ${ }^{127} \mathrm{I}$ and ${ }^{74} \mathrm{Ge}$, respectively, for DAMA and CoGeNT, as their detectors are made of $\mathrm{NaI}$ and Ge crystals. The choice of the iodine component of the DAMA/LIBRA experiment, rather than ${ }^{23} \mathrm{Na}$, is crucial since it allows getting rid of the formation of bound states with light elements, thus preventing the formation of anomalous heavy isotopes on Earth and during Big Bang nucleosynthesis. A direct consequence is that the collisions in the terrestrial crust are purely elastic.

For each set of parameters and for each experiment, the Schrodinger equation independent of time, with potential $V_{k}^{\text {nucl }}+V_{m}^{\text {nucl }}$ applied to the constituent nucleus, is first solved through the WKB approximation. This gives good approximations for the eigenvalues and eigenfunctions of the corresponding nucleus- $F$ systems, the former allowing us to calculate $\Delta E$. The modulated part $n_{F}^{m}$ of the number density of $F$ in the detector is then computed using (17) before finally evaluating the modulated part of the event rate $R^{m}$ from (22), at the operating temperatures $T=300 \mathrm{~K}$ for DAMA and $T=$ $77 \mathrm{~K}$ for CoGeNT. To compute the capture cross section $\sigma_{\text {capt }}$, 


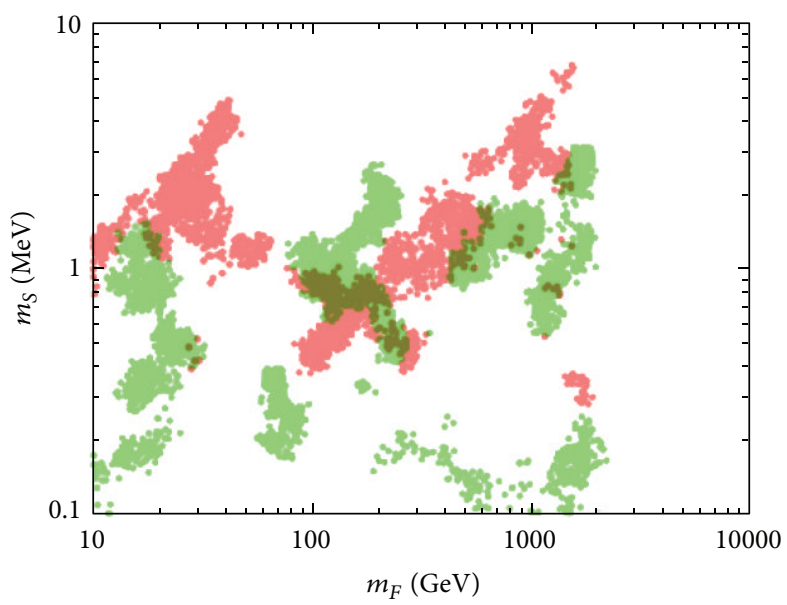

(a)

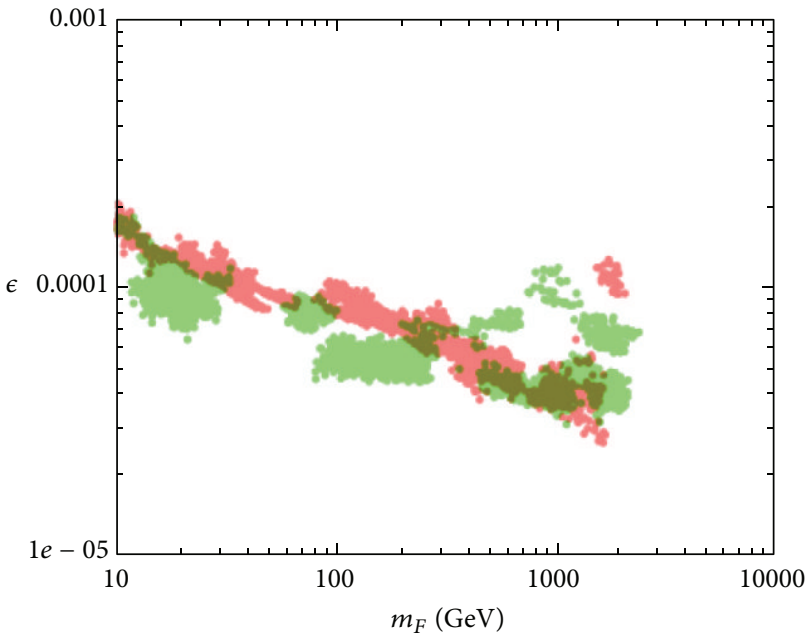

(c)

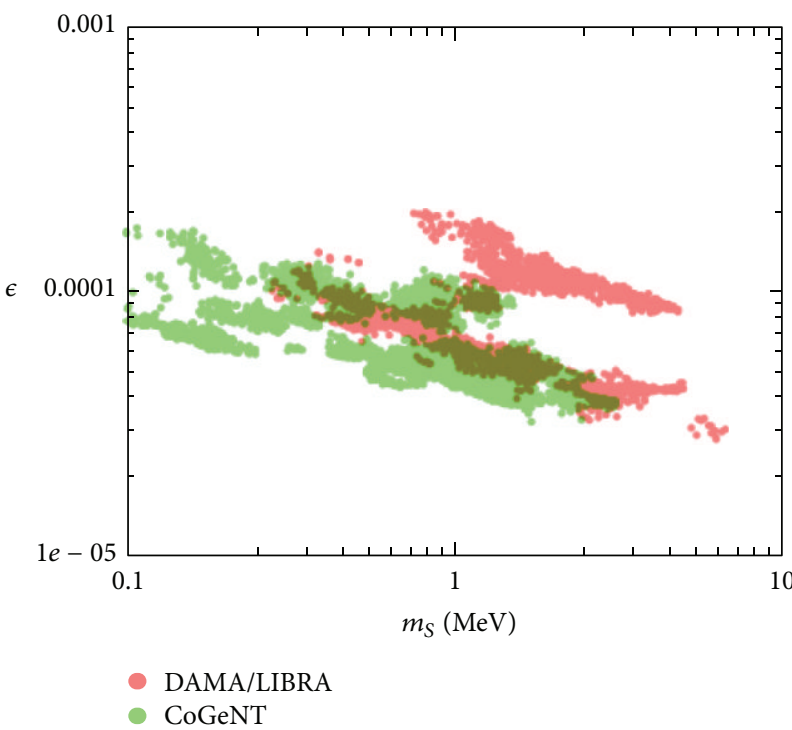

(e)

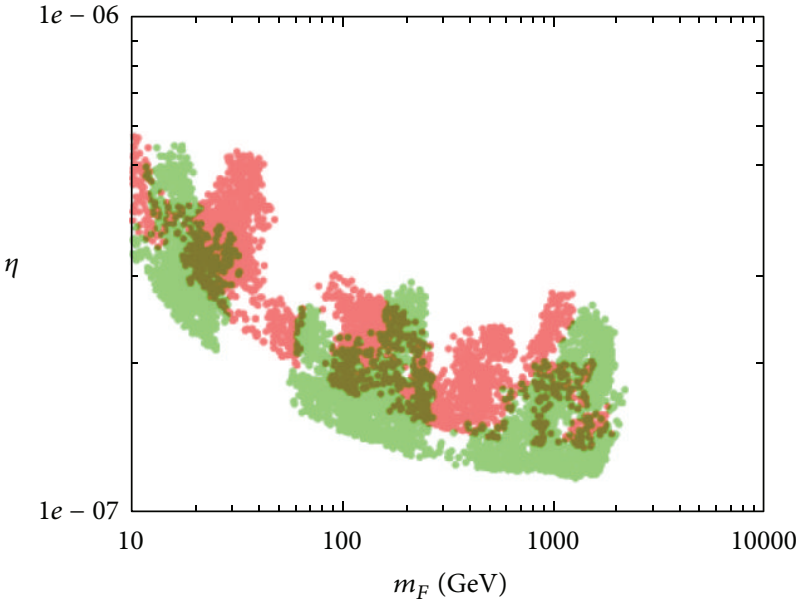

(b)

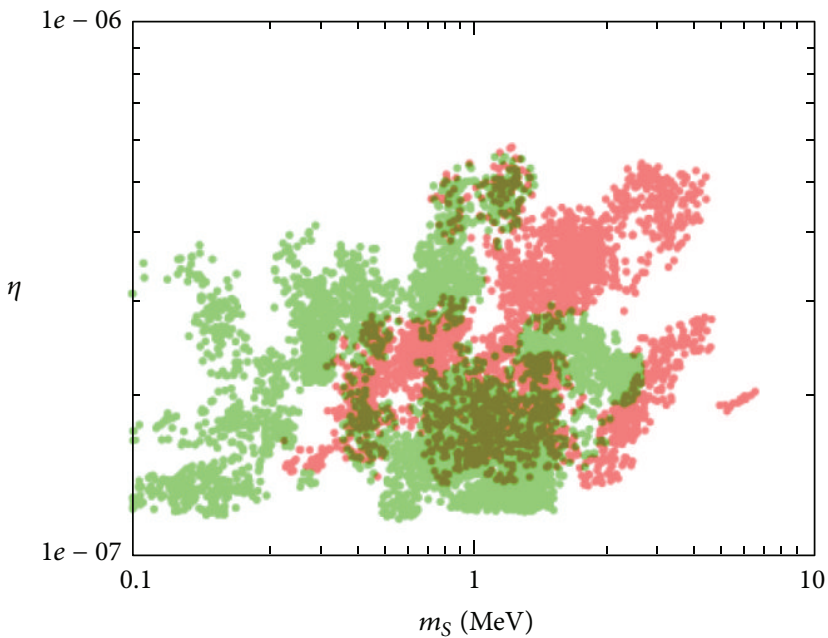

(d)

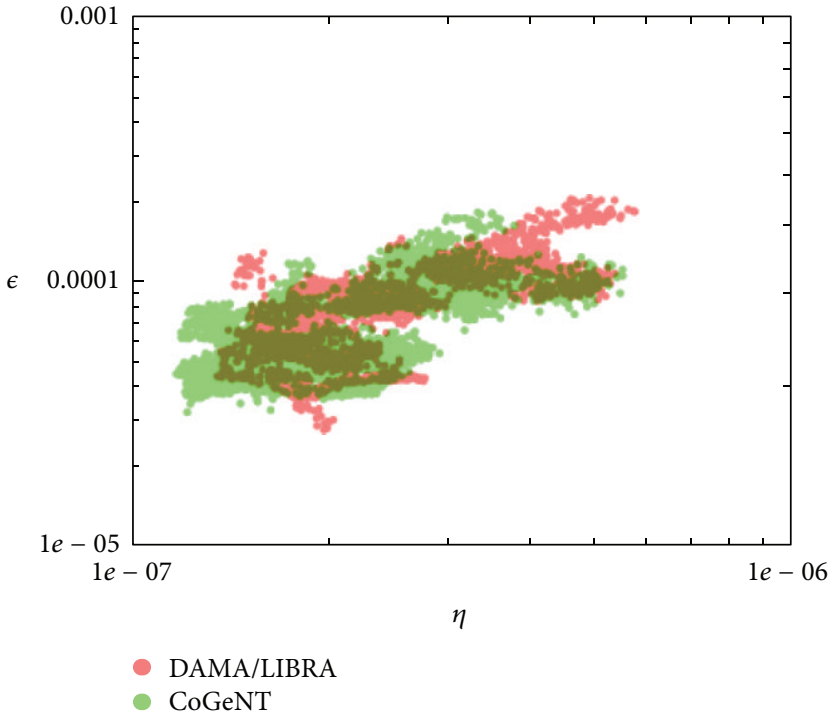

(f)

FIGURE 1: Two-dimensional parameter regions reproducing the DAMA/LIBRA (light red) and CoGeNT (light green) results at the $2 \sigma$ level. The overlapping regions stand out in dark green. (a) $\left(m_{F}, m_{S}\right)$ plane. (b) $\left(m_{F}, \eta\right)$ plane. (c) $\left(m_{F}, \epsilon\right)$ plane. (d) $\left(m_{S}, \eta\right)$ plane. (e) $\left(m_{S}, \epsilon\right)$ plane. (f) $(\eta, \epsilon)$ plane. 
given by (18), at a given energy $E$ in the center-of-mass frame of the nucleus- $F$ system, one numerically solves the radial Schrodinger equation in the continuum to get the radial part $R(r)$ of the initial diffusion eigenstate and calculate the matrix element $D$ of the electric dipole operator.

The regions are projected in two dimensions by combining all the possible pairs of parameters and are given in Figure 1. For each model, one has ensured that the first emitted photon has an energy below the threshold of the considered experiment while the second one has an energy beyond the threshold, that thermalization occurs before $1 \mathrm{~km}$, that no bound states can form with elements characterized by $Z \leq 14$ ( $Z=14$ being silicon), and that thermalization at the edge of the CoGeNT detector requires a penetration length much shorter than the size of the detector. For the latter point, we have used (13) and (14) with $E_{0}=(3 / 2) T_{\text {room }}$, where $T_{\text {room }}=300 \mathrm{~K}$ is the initial room temperature, and $E_{\mathrm{th}}=(3 / 2) T$, where $T=77 \mathrm{~K}$ is the final temperature.

From the overlapping regions in the projected parameter spaces implying $m_{F}$, we see that possible values for that parameter are between $10 \mathrm{GeV}$ and $2 \mathrm{TeV}$. The upper limit comes from the requirement that the penetration length must be less than $1 \mathrm{~km}$. Analyzing the regions where the parameter $m_{S}$ is involved indicates that the values reproducing both the DAMA/LIBRA and CoGeNT experiments at the $2 \sigma$ level must lie within $[0.4,3] \mathrm{MeV}$. In the same way, we find that $\eta$ ranges from $1.3 \times 10^{-7}$ to $5 \times 10^{-7}$ while $\epsilon$ goes from $3 \times 10^{-5}$ to $2 \times 10^{-4}$.

5.2. Considerations about the Constraints on $\eta$ and $\epsilon$. One has derived, in [19], a constraint on $\tilde{\eta}=\eta g / g^{\prime}$ from unseen vector meson disintegrations: $\widetilde{\eta}<1.2 \times 10^{-4}$. In principle, it is not applicable to $\eta$, but a reasonable choice would consist in posing $g^{\prime}=g$. In this case, the constraint translates directly to $\eta$ and we see that all the previous models satisfy it easily, by two or three orders of magnitude.

The cosmological and astrophysical constraints on $\epsilon$, generally derived in the framework of models with a single millicharged species realizing the full cosmological dark matter density, cannot be applied directly to this subdominant, atomic, and millicharged scenario and should in any case be somewhat weakened. However, constraints from accelerators can always be used. For masses $m_{F} \geq 1 \mathrm{GeV}$, they let a large allowed window for $\epsilon<0.1$ [24], which is clearly the case here. Some interesting discussion may arise from the lighter species $G$, with constraints on $\epsilon$ from accelerators being stronger for smaller masses. Similarly to $\eta, m_{G}$ is not directly constrained by the previous analysis but only the product of $m_{G}$ and $e^{\prime 2}$ through the Bohr radius $a_{0}^{\prime}$ of the dark atoms. However, if we do once again the reasonable assumption $e^{\prime} \simeq e$, then the adopted value of $a_{0}^{\prime}=1 \AA$ leads to $m_{G} \simeq m_{e}$, where $m_{e}$ is the mass of the electron. It turns out that for $m_{G} \sim 1 \mathrm{MeV}$, the upper limit on $\epsilon$ from accelerators lies just in the interval deduced in the previous section from direct experiments. If it is so, we could therefore be close to a discovery of millicharges in accelerators via the component G.
5.3. Consistency with XENON100, CDMS-II/Ge, and LUX. For the models of Figure 1 to be fully acceptable, we have to ensure that they satisfy the constraints set by the experiments that do not observe any signal, as XENON100, CDMSII/Ge, and LUX. These are able to discriminate between nuclear and electron recoils and, as already mentioned at the end of Section 4.3, bound-state-formation events producing electron recoils in such detectors will be considered as backgrounds. Therefore, if some events remain, they should still have a smaller rate than the observed background.

XENON100 and LUX have similar detectors, but LUX puts the strongest constraint with expected and observed electron-recoil backgrounds, respectively, of $\left(2.6 \pm 0.2_{\text {stat }} \pm\right.$ $\left.0.4_{\text {syst }}\right) \times 10^{-3}$ and $\left(3.1 \pm 0.2_{\text {stat }}\right) \times 10^{-3} \mathrm{cpd} / \mathrm{kg} / \mathrm{keV}_{e e}$ in the $(0.9-5.3) \mathrm{keV}_{e e}$ range. This leaves the possibility of an additional contribution to the expected background of at most $5.72 \times 10^{-3} \mathrm{cpd} / \mathrm{kg}$ in that energy interval. Computing the constant part $R^{0}$ of the rate from (22) for ${ }^{132} \mathrm{Xe}$ and at the operating temperature $T=173 \mathrm{~K}$, and rejecting the models leading to higher rates, does not change the ranges of parameters previously found from the reproduction of the experiments with positive results.

Finally, this model predicts strongly suppressed event rates in cryogenic detectors, such as CDMS-II, where temperatures $\sim 1 \mathrm{mK}$ give rise to much too low thermal energies for the dark atoms to tunnel through the Coulomb barrier and be captured. The rates computed with ${ }^{74} \mathrm{Ge}$ at $T=1 \mathrm{mK}$ are effectively consistent with zero and are therefore in agreement with the negative results from CDMS-II/Ge.

\section{Conclusions}

We have explored the parameter space of our milliinteracting dark matter model and found, while only one model was given in [19], that regions reproducing the directdark-matter-search experiments at the $2 \sigma$ level can be identified. The overlaps of the regions of DAMA/LIBRA and CoGeNT indicate that the interesting models must lie in the ranges $10 \mathrm{GeV} \leq m_{F} \leq 2 \mathrm{TeV}, 0.4 \mathrm{MeV} \leq m_{S} \leq 3 \mathrm{MeV}$, $10^{-7} \leq \eta \leq 5 \times 10^{-7}$, and $3 \times 10^{-5} \leq \epsilon \leq 2 \times 10^{-4}$. Within these intervals, models that do not contradict the negative results from XENON100 and LUX exist, and their rates contribute to the expected electron-recoil background. The model naturally prevents any bound-state-formation event in cryogenic detectors $(T \sim 1 \mathrm{mK}$ ), which is in agreement with the Germanium detector of CDMS-II. Some difficulties appear, however, with the CRESST-II and CDMSII/Si cryogenic experiments, for which the collisions at the edges of the detectors should be studied in detail, when the particles are still at room temperature and can have sufficient energies to be captured and produce a signal.

More than giving constraints on the parameters of a specific model, it has been shown here that it is possible, in the framework of dark matter models containing a sector with a richness and a complexity similar to ours, to reconcile experiments such as DAMA/LIBRA and XENON100 that seem contradictory when interpreted in terms of WIMPs. 


\section{Conflict of Interests}

The author declares that there is no conflict of interests regarding the publication of this paper.

\section{Acknowledgments}

The author thanks J. R. Cudell for useful advice. This work is supported by the Belgian fund F.R.S-FNRS.

\section{References}

[1] R. Bernabei, P. Belli, F. Cappella et al., "New results from DAMA/LIBRA," The European Physical Journal C, vol. 67, no. 1, pp. 39-49, 2010.

[2] R. Bernabei, P. Belli, F. Cappella et al., "Final model independent result of DAMA/LIBRA-phase1," The European Physical Journal $C$, vol. 73, article 2648, 2013.

[3] C. E. Aalseth, P. S. Barbeau, J. Colaresi et al., "Search for an annual modulation in a $p$-type point contact Germanium dark matter detector," Physical Review Letters, vol. 107, no. 14, Article ID 141301, 5 pages, 2011.

[4] J. Collar, "Search for an annual modulation in 3.4 years of CoGeNT data," in Proceedings of the 13th International Conference on Topics in Astroparticle and Underground Physics (TAUP '13), Asilomar, Calif, USA, September 2013.

[5] G. Angloher, M. Bauer, I. Bavykina et al., "Results from $730 \mathrm{~kg}$ days of the CRESST-II Dark Matter search," The European Physical Journal C, vol. 72, no. 4, article 1971, pp. 1-22, 2012.

[6] R. Agnese, Z. Ahmed, A. J. Anderson et al., "Silicon Detector Dark Matter Results from the Final Exposure of CDMS II," Physical Review Letters, vol. 111, no. 25, Article ID 251301, 6 pages, 2013.

[7] E. Aprile, M. Alfonsi, K. Arisaka et al., "Dark matter results from 225 live days of XENON100 data," Physical Review Letters, vol. 109, no. 18, Article ID 181301, 6 pages, 2012.

[8] Z. Ahmed, D. S. Akerib, S. Arrenberg et al., "Results from a low-energy analysis of the CDMS II Germanium data," Physical Review Letters, vol. 106, no. 13, Article ID 131302, 5 pages, 2011.

[9] D. S. Akerib, H. M. Araujo, X. Bai et al., "First results from the LUX dark matter experiment at the Sanford Underground Research Facility," 2013.

[10] R. Foot, "Mirror dark matter interpretations of the DAMA, CoGeNT and CRESST-II data," Physical Review D, vol. 86, no. 2, Article ID 023524, 10 pages, 2012.

[11] J. M. Cline, Z. Liu, and W. Xue, "Millicharged atomic dark matter," Physical Review D, vol. 85, no. 10, Article ID 101302, 6 pages, 2012.

[12] M. Y. Khlopov, A. G. Mayorov, and E. Y. Soldatov, "The dark atoms of dark matter," Prespacetime Journal, vol. 1, pp. 1403-1417, 2010.

[13] M. Y. Khlopov, A. G. Mayorov, and E. Y. Soldatov, "Towards nuclear physics of $\mathrm{OHe}$ dark matter," in Proceedings of the 14th International Workshop, "What Comes Beyond the Standard Model”, N. Mankoc Borstnik, H. B. Nielsen, C. D. Froggatt, and D. Lukman, Eds., vol. 12, pp. 94-102, Bled, Slovenia, 2011.

[14] J. R. Cudell, M. Khlopov, and Q. Wallemacq, "The nuclear physics of OHe," in Proceedings of the 15th International Workshop "What Comes Beyond the Standard Model", N. Mankoc Borstnik, H. B. Nielsen, and D. Lukman, Eds., vol. 13, pp. 1027, Bled, Slovenia, 2012.
[15] M. McCullough and L. Randall, "Exothermic double-disk dark matter," Journal of Cosmology and Astroparticle Physics, vol. 2013, 2013.

[16] N. Fornengo, P. Panci, and M. Regis, "Long-range forces in direct dark matter searches," Physical Review D, vol. 84, no. 11, Article ID 115002, 20 pages, 2011.

[17] J. Miralda-Escudé, "A test of the collisional dark matterhypothesis from cluster lensing," The Astrophysical Journal, vol. 564, no. 1, pp. 60-64, 2002.

[18] J. Fan, A. Katz, L. Randall, and M. Reece, "Double-disk dark matter," Physics of the Dark Universe, vol. 2, no. 3, pp. 139-156.

[19] Q. Wallemacq, "Milli-interacting dark matter," Physical Review $D$, vol. 88, no. 6, Article ID 063516, 10 pages, 2013.

[20] C. Amsler, M. Doser, M. Antonelli et al., "Review of particle physics," Physics Letters B, vol. 667, no. 1-5, pp. 1-6, 2008.

[21] G. Erkol, R. G. E. Timmermans, and T. A. Rijken, "Nucleonsigma coupling constant in QCD sum rules," Physical Review C, vol. 72, no. 3, Article ID 035209, 7 pages, 2005.

[22] S. D. McDermott, H. B. Yu, and K. M. Zurek, "Turning off the lights: how dark is dark matter?" Physical Review D, vol. 83, no. 6, Article ID 063509, 9 pages, 2011.

[23] E. Segre, Nuclei and Particles, W. A. Benjamin, New York, NY, USA, 2nd edition, 1977.

[24] J. Jaeckel and A. Ringwald, "The low-energy frontier of particle physics," Annual Review of Nuclear and Particle Science, vol. 60, pp. 405-437, 2010. 

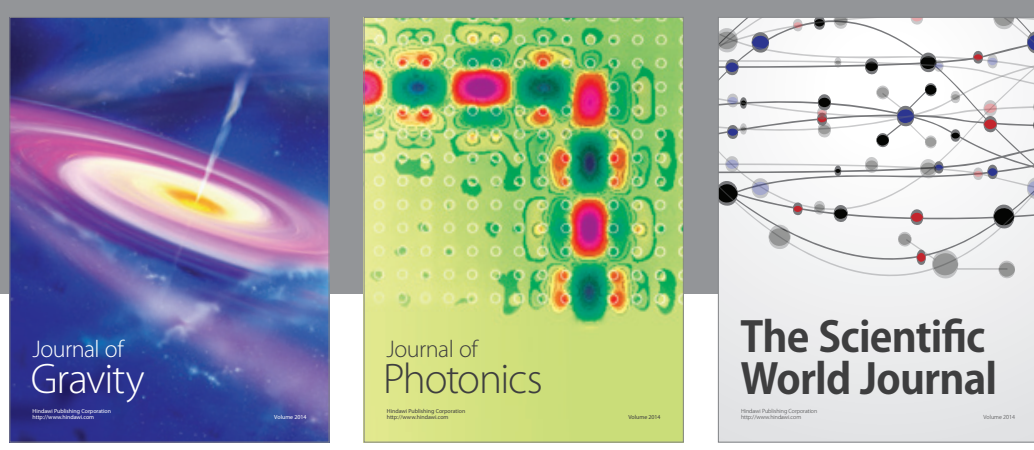

The Scientific World Journal
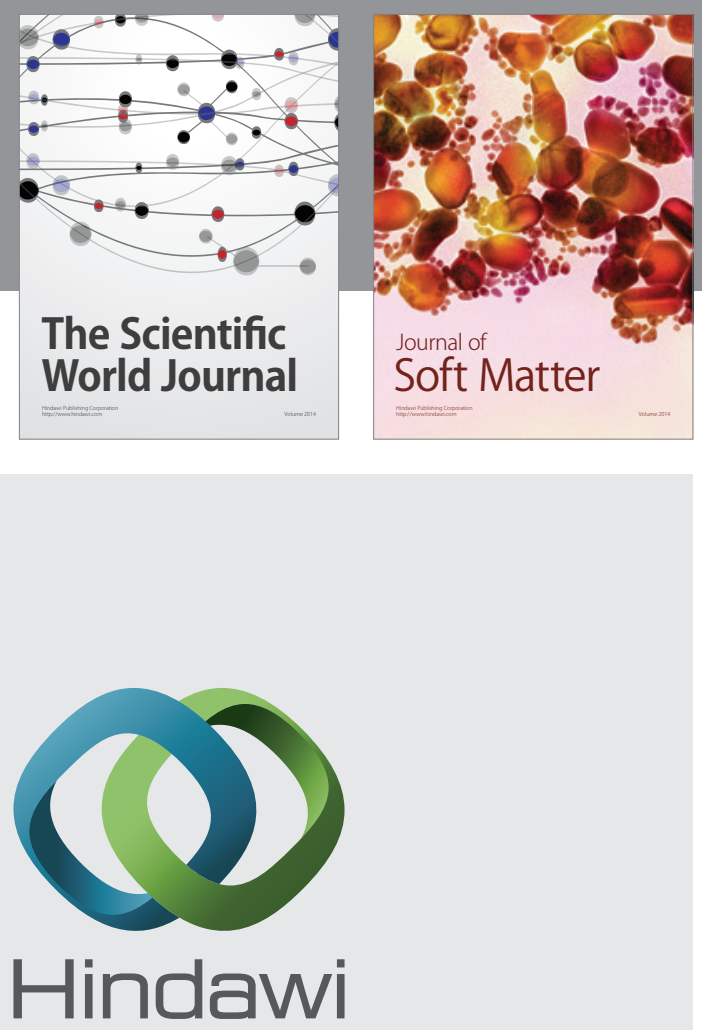

Submit your manuscripts at

http://www.hindawi.com

nternational Journal of

Statistical Mechanics
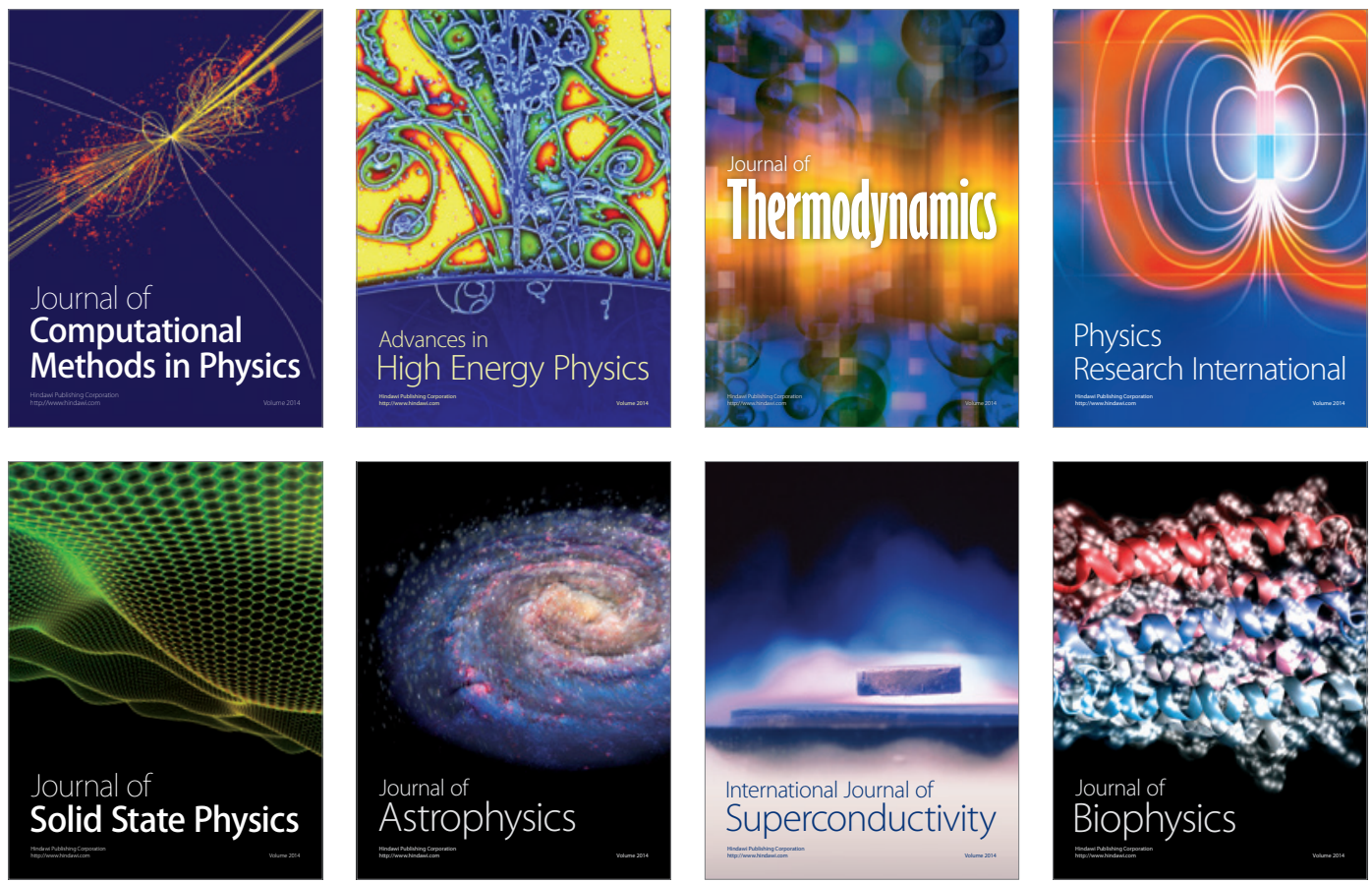
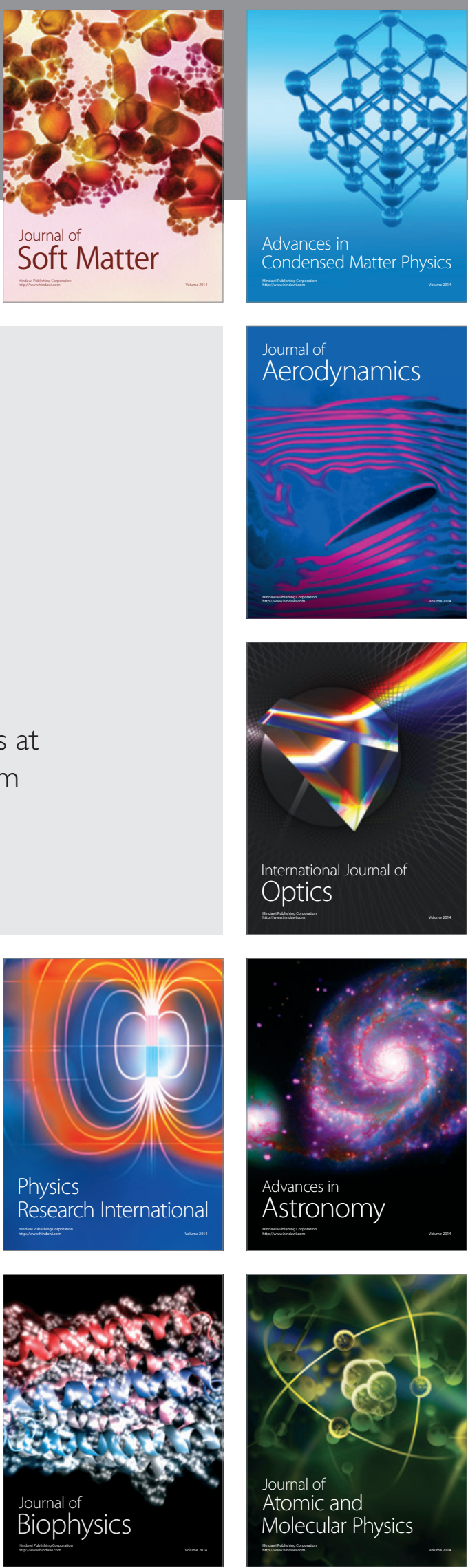